\title{
Tactual perception: a review of experimental variables and procedures
}

\author{
Alexandra M. Fernandes • Pedro B. Albuquerque
}

Received: 1 February 2012/ Accepted: 18 May 2012/Published online: 6 June 2012

(C) Marta Olivetti Belardinelli and Springer-Verlag 2012

\begin{abstract}
This paper reviews the literature on tactual perception. Throughout this review, we will highlight some of the most relevant aspects in the touch literature: type of stimuli; type of participants; type of tactile exploration; and finally, the interaction between touch and other senses. Regarding type of stimuli, we will analyse studies with abstract stimuli such as vibrations, with two- and threedimensional stimuli, and also concrete stimuli, considering the relation between familiar and unfamiliar stimuli and the haptic perception of faces. Under the "type of participants" topic, we separated studies with blind participants, studies with children and adults, and also performed an overview of sex differences in performance. The type of tactile exploration is explored considering conditions of active and passive touch, the relevance of movement in touch and the relation between haptic exploration and time. Finally, interactions between touch and vision, touch and smell and touch and taste are explored in the last topic. The review ends with an overall conclusion on the state of the art for the tactual perception literature. With this work, we intend to present an organised overview of the main variables in touch experiments, compiling aspects reported in the tactual literature, and attempting to provide both a summary of previous findings, and a guide to the design of future works on tactual perception and memory, through a presentation of implications from previous studies.
\end{abstract}

Keywords Tactual perception - Review $\cdot$ Haptics $\cdot$ Touch

A. M. Fernandes $(\bowtie) \cdot$ P. B. Albuquerque

School of Psychology, University of Minho, Campus de Gualtar, 4710-057 Braga, Portugal

e-mail: alexandra.fernandes@gmail.com
Touch is relevant in everyday tasks, and haptic memory allows us to remember the feeling of objects and the touch of other people and is crucial in our everyday life. From picking a coin from our wallet to evaluating the force we need to apply to open a door, we rely on touch in various everyday tasks. Touch has been a relatively unattended sensory modality in cognitive research. Some studies have developed research on touch, but most of the touch literature is driven by perception research, attempting to compare touch with other sensory modalities.

When we think about touch, we have to comprehend it as a complex system with more than one sense: touch involves pain perception, temperature perception, proprioception, kinaesthetic perception and tactile perception. In the touchrelated literature, the concepts tactile, haptic and tactual are common and are often presented as synonymous, although they have been defined as distinct concepts previously (e.g., Loomis and Lederman 1986). In the present work, we will use them as distinct concepts. Tactile perception refers to the extraction of material properties from the stimuli, such as texture, that are distinct from geometrical properties, such as shape, that usually require a more active interaction with the objects (Lederman and Klatzky 2009). Tactile perception results from cutaneous variations only (Loomis and Lederman 1986) and is associated with situations of passive touch, in which the participants cannot explore the objects, keeping their hands or fingers still while the stimuli are pressed against their skin. However, most stimulus properties are extracted through a combination of cutaneous and movement cues; this combination results in more than just a sum of kinaesthetic and tactile inputs, in the sense that only a complex integration of these cues allows the perception of objects in space and in relation to each other (Kaas et al. 2008). This type of perception through touch is called haptic perception (Loomis and Lederman 1986). Haptic perception is the most natural 
form of perception through touch and is more related with free exploration procedures. Finally, the term "tactual perception" is a more generic concept and refers to all types of perception derived from cutaneous (tactile) and kinaesthetic (movement) cues and is used to describe overall perception by touch, regardless of experimental conditions and can be assumed as a synonym of touch.

This literature review aims to contribute to the systematisation of information about touch by analysing the main variables manipulated within perceptual paradigms, namely the interactions of touch with other senses, the type of stimuli, the type of participants and the tactile exploratory conditions.

\section{Type of stimuli: abstract stimuli}

A critical review of studies with abstract stimuli will be presented. Abstract stimuli are here defined as uncommon and unnameable stimuli that are not usually available in our everyday experience and are frequently created in the laboratory in order to evaluate specific features of tactual perception and cognition. This section is divided in two parts: first, studies with vibration stimuli and second, studies using two-dimensional and three-dimensional stimuli are reported.

\section{Vibrations}

The use of vibrating stimuli in tactile research has been common in the definition of tactile thresholds (e.g., Hagander et al. 2000), tactile masking paradigms (e.g., Craig and Evans 1987) or tactile pattern perception (e.g., Cholewiak et al. 2001). Vibrations have also been studied under more applied areas, for instance the use of vibration as warning signals in driving (e.g., Ho et al. 2006; Spence and Ho 2008). Also, the perception of vibration has allowed the development of communication systems for sensorydeprived subjects, such as the Tadoma system, a system by which deaf-blind subjects are able to perceive speech by placing their hands on the speakers face and feeling the vibrations (e.g., Sherrick 1975).

Vibration is useful in the perception of roughness, and Katz (1925/1989) mentioned the involvement of the sense of vibration in tactile perception. Lederman et al. (1982) confirmed the relevance of vibration in roughness perception and argued that vibration allowed continuous activation of the mechanoreceptors of the skin, facilitating texture perception.

Vibrotactile stimulation has also been used within working memory paradigms. For instance Bancroft and Servos (2011), using a matching to sample paradigm, explored interference effects in working memory.
Participants had to determine whether two separate vibrotactile stimuli had the same frequency or not. Between the two stimuli, an interfering distractor was introduced. Results showed that the frequency of the distractor affected performance, with trials in which the distractor was more similar to the probe having more errors than trials in which the distractor was more similar to the target. The authors assume that the distractor was overwriting the probe stimuli and argue in favour of an overwriting system to explain interference effects in working memory.

The use of vibrotactile stimuli, although more common in physiological (e.g., Verrillo 1968) or psychophysical (e.g., Verrillo and Gescheider 1975) procedures, is also reported in perception and memory research, allowing the study of touch within controlled exposition conditions. Vibrations have the advantage of being a highly controlled stimuli in touch since the experimenters can strictly define where the vibration is applied, when the vibration is applied, its frequency, the size of the stimulated area and the duration of the stimulation.

Two-dimensional and three-dimensional stimuli

Aiming to evaluate tactual perception and cognition in isolation, many researchers have chosen to collect data using artificial stimuli created in the laboratory. These stimuli, either two- or three-dimensional, allow absolute control of stimuli properties and are very useful to understand how people discriminate single features of the objects, or how different properties are weighted and integrated.

Regarding two-dimensional stimuli, two main types of stimuli can be found: raised line drawings or haptic pictures, which consist of the presentation of the contours of a drawing in a higher level to the background; and tactile scenes, presentation of matrices or other complex stimuli that can only be explored in two dimensions.

Plaisier et al. (2008) have explored the pop-out effect on haptics. The pop-out effect has been studied in vision and refers to the easier detection of a stimulus among others in a visual search: for instance, detecting a red stimulus among green ones. In this first study on haptic pop-out (Plaisier et al. 2008), it was shown that a rough item among fine items in a matrix was detected faster than the inverse, demonstrating the existence of a pop-out effect in haptics. Analysing bilateral symmetry, Ballesteros et al. (1997) reported that for raised line shapes, perception of asymmetry was better than perception of symmetry when participants could explore the item with only one finger and that bimanual exploration facilitated symmetry judgments, but not asymmetry ones. For unfamiliar three-dimensional stimuli, single hand and bimanual explorations did not differ, and symmetry judgments were very accurate. 
The variability of three-dimensional abstract stimuli is higher than in two-dimensional stimuli, and these types of stimuli can be geometrical or abstract forms built in various materials (e.g., plastic, wood, metal) or stimuli created from the combination of toy construction blocks.

Kiphart et al. (1988) used three-dimensional uncommon (hardly nameable) geometrical plastic shapes (stimuli from the "Superfection" game) and reported a hit rate of $93 \%$ in haptic recognition, with false alarms being virtually nonexistent even for delays as large as $40 \mathrm{~s}$ between item presentation and recognition for a total of 30 objects. In a later study (Kiphart et al. 1992), the authors reported high discriminability measures, even when the stimuli were attached to a base, and the participants only had $3 \mathrm{~s}$ to explore them.

Regarding the discrimination of Gaussian-like shapes, attending to amplitude and width parameters Louw et al. (2002) showed that participants were able to haptically discriminate up to 300 shapes, being better at discriminating sharp from smooth Gaussian curves than at discriminating between small and large ones. The authors concluded that the estimation of width was central for the discrimination of shape.

Using three-dimensional objects, van der Horst et al. (2008) explored the effect of curvature in haptic perception. The participants were asked to differentiate cylinders with a circular versus an elliptical base and to discriminate square from rectangular cuboids. Results showed that the base ratio to perceive objects with curvature was smaller than the needed ratio to perceive cuboid differences, suggesting that curvature is a relevant cue in haptic experience, facilitating object perception.

According to Klatzky et al. (1985), this group of studies on touch using abstract stimulation, might be contributing to an underestimation of haptic abilities. To these authors, a main distinction between abstract and everyday objects' perception and recognition rises from the concept of pattern recognition. Everyday objects are perceived as patterns and analysed as a whole, while abstract stimuli are perceived as a sum of different features (Klatzky and Lederman 2003). They argue that haptic recognition of everyday objects entails an apprehension of tactile patterns that is achieved through both bottom-up and top-down processes, and these aspects differentiate haptic perception from the simple categorisation that is typical in the perception of abstract stimuli (Klatzky et al. 1985).

Experiments with two- and three-dimensional abstract stimuli are very useful to analyse haptic evaluation of specific properties, allowing an estimation of the impact of each tactual feature in the overall perception of the stimuli, and as such contributing to a deeper understanding of how people perceive and process everyday tactual stimuli.

This section presented an overall scenario of research with abstract stimuli, showing that these type of stimuli contribute to a detailed analysis of tactual processes, allowing for a greater control of experimental conditions, such as manipulation times or stimuli variability. However, these techniques remove crucial cues from haptics such as material properties (e.g., texture or weight information), and as such might be resulting in an estimation of tactual ability that does not necessarily correspond to performance in optimal conditions with free manipulation and complex objects. The following section will explore performance with concrete stimuli.

\section{Type of stimuli: concrete stimuli}

This section regards performance with concrete stimuliusually three-dimensional and common stimuli-evaluating differences between familiar and unfamiliar stimuli and more recent topic: the tactual perception of faces.

Familiar and unfamiliar stimuli

Bülthoff and Newell (2006) presented a thorough review on the role of familiarity in the perception of objects, faces and movement. The authors concluded that stimulus familiarity is a central feature in perception and that people are more efficient responding to learned than unknown objects, which led the authors to assume that our perceptual system is organised through these familiar stimuli or events.

A study with 5-year-old children showed that they were very good at haptically recognising a list of 16 unfamiliar objects (e.g., segments of other objects), and their performance was optimal in the familiar objects' set, with children being able to correctly recognise about 15 out of 16 presented objects (Bushnell and Baxt 1999). Also Lacey and Campbell (2006), in a study with adults in a crossmodal visuo-haptic paradigm, reported that participants were able to recognise between 15 and 16 objects in a 16-object set for familiar items and around 13-14 objects in unfamiliar object trials. In two recent functional magnetic resonance studies (Lacey et al. 2010; Deshpande et al. 2010), the authors argued that crossmodal visuo-tactile interactions in the brain are modulated by the item's familiarity, when they found that the activation overlapping between haptic and visual trials was bigger for familiar than unfamiliar objects.

A series of studies has also shown that people have preferred "views" or perspectives to perceive objects by touch. These views are called canonical views and had been studied in vision, with people being more effective at recognising objects presented from a familiar perspective than when presented in a different orientation (Newell et al. 2001). Under free exploration conditions, participants still 
preferred the haptic canonical view to manipulate objects and the hands favoured a "back" perspective instead of the "front" perspective, typical of vision (Newell et al. 2001). Participants showed coherence in the selection of haptic canonical views, either for familiar or for unfamiliar stimuli, and these perspectives allowed a better recognition performance (Woods et al. 2008). The viewpoint dependency in touch is verified either for stimuli learned in the experimental task or for everyday stimuli and is mediated by task difficulty, with the most difficult discrimination tasks being more impaired by changes in stimuli perspective (Lawson and Bulthoff 2008). Even within the viewpoint or orientation procedures, differences can be found between familiar and unfamiliar objects (Craddock and Lawson 2008), with objects' recognition being dependent on the orientation in which stimuli were presented, for both types of objects, but with larger errors for unfamiliar than for familiar objects.

Haptic recognition of familiar objects is also sensitive to size and shape changes (Craddock and Lawson 2009b). In this study, participants were asked to recognise objects based on identity and ignoring size or shape variations. Results showed that generalisation occurred in touch, but there was a cost, since participant's recognition performance was lightly impaired in trials in which a change was present.

Klatzky et al. (1985) had shown touch to be an effective and highly accurate system in the perception of familiar everyday objects and had defined that people need about two to $3 \mathrm{~s}$ (sometimes less) to be able to identify a stimulus presented through touch only. A later work (Klatzky and Lederman 1995) revealed that participants were able to identify familiar objects without any cues with presentations as short as $200 \mathrm{~ms}$, with an accuracy level of $20 \%$. When analysing the errors, the authors found that the most typical errors corresponded to identifications of other items similar to the target (e.g., identifying a pencil as a pen).

Familiarity is a central variable in tactual cognition, and the ability of the haptic system in recognition and perception of these stimuli must be explored, since it can provide valuable information to the understanding of touch.

\section{Faces}

The representation of faces has been considered distinct from the representation of other stimuli (Kanwisher 2000). Most studies on face perception and recognition have been conducted within the visual modality (e.g., Bruce and Young 1998), but in recent years, the study of haptic facial recognition has produced interesting results. Kilgour and Lederman (2002) reported a study with three conditions: haptic study and recognition of live faces, haptic study and recognition of facial masks, and visuo-haptic study of live faces with haptic recognition. Results showed that participants were able to correctly identify unfamiliar faces by touch only with rates of about $80 \%$ for the first condition, about $70 \%$ in the second and about $60 \%$ in the last. The last two conditions with haptic recognition of facemasks implied a loss of material cues of the stimuli and participants had to perform their evaluations based on geometrical cues only. Since material cues are central in haptic recognition (Klatzky et al. 1987), this is an expected result.

Also, people can recognise facial emotional expressions by touch only, whether touching facemasks or people interpreting the emotional expression (Lederman et al. 2007). In the same study, haptic recognition of emotional facial expressions was analysed comparing dynamic (expressions made by actors) and static (masks representing emotions) emotional faces. The hypothesis was that if participants can recognise the haptic and tactile inputs of an emotional face, they should be able to correctly identify the six universal expressions of emotion (Lederman et al. 2007). Results revealed that participants were better at recognising dynamic than static emotional expressions and that happiness, sadness and surprise were easier to recognise by touch than anger, disgust and fear.

Casey and Newell (2005) studied familiarity in haptic recognition of faces and concluded that participants were not able to haptically recognise their own face, although visual priming of their live face facilitated the task. In a second experiment, the authors reported that familiarisation with a group of unknown faces improved crossmodal recognition (Casey and Newell 2005). In a later study (Casey and Newell 2007), the authors concluded that face representations were modality-specific, although configurational properties of the faces seemed to be shared between vision and touch (in opposition to feature information).

The relevance of familiarity in face perception has also been pointed out by Bülthoff and Newell (2006), arguing that familiarity is a central process in human perception and that familiar stimuli always present better learning and recognition performance.

The haptic system appears to be a valuable perceptual resource, yielding surprisingly good performance even in tasks that are not familiar or recurrent in everyday experience, such as recognising a face or identifying an emotional expression through touch only.

Object familiarity is a crucial dimension for touch as well as for other sensory modalities, confirming the familiarity advantage as a general principle of cognitive processing. As shown, familiarity produces enhanced performances, even in unfamiliar tasks, as the haptic recognition of a face or a facial expression of emotion. The distinction between tasks with familiar and unfamiliar stimuli also translates this tendency to find patterns and to organise and process stimuli according to previous 
cognitive rules and schemas in touch as well as in other sensory modalities.

\section{Type of participants}

Next, we will present a set of experiments according to the type of participants that were selected. In tactual research, blind participants have been a relevant group, generating an interesting amount of results; next, we will present results obtained in studies with children and infants, and finally, we will evaluate the relevance of considering sex differences in tactual performance.

\section{Blind participants}

Historically, the study of touch and blindness has roots in philosophy, medicine and psychology. The idea of enhanced tactile and auditory perception in blind subjects (e.g., Cattaneo and Vecchi 2011) and the notion of sensory substitution (e.g., Bach-y-Rita 1972) are common in the literature. However, research shows that information from different sensory modalities is not always easily interchangeable (e.g., Röder and Rösler 2004).

Authors have considered the study of haptic perception with blind participants as relevant because studies with sighted participants might be resulting in an underestimation of haptic abilities (Heller and Ballesteros 2006), since there are reports of enhanced haptic sensitivity in the blind (e.g., Sathian and Prather 2006). Research with blind participants has suggested that they have better performance in haptic tasks than sighted participants, for instance blind participants were faster in a task that required matching of geometrical shapes to its cut-offs (Postma et al. 2007), but this advantage is not always present. In a study considering the discrimination of texture, grating orientation and vibrotactile frequency, blind participants showed an advantage only for texture discrimination, revealing equivalent performance to sighted participants in the other two tasks (Alary et al. 2009).

Many authors have emphasised the role of practice in performance for touch (e.g., Cohen et al. 2011), and within this perspective, one must consider that blind participants have a larger experience in perceiving objects mainly by touch than sighted participants, and this familiarisation might be modulating the results, at least for experiments in which the stimuli are common or familiar stimuli.

However, Abramowicz et al. (2010) have shown that with the presentation of highly unfamiliar and uncommon stimuli, blind and sighted participants show equivalent learning rates. In this study, the authors showed that it was possible to perceive emotional facial expressions through schematic raised line drawings. Another central conclusion of this study was that blind participants could be trained to detect emotional facial expressions. Also, in a study, comparing performance in haptic recognition of twodimensional patterns with early-blind, late-blind and sighted participants, it was described that the three groups had similar rates of performance, although participants were reporting different memory strategies when encoding the stimuli (Picard et al. 2010).

The comparison of blind and sighted participants in an angle discrimination task showed better performance of the blind participants' group (Alary et al. 2008). Participants had to touch two-dimensional angles with one finger and evaluate which one was the largest. Blind participants consistently revealed lower thresholds for angle discrimination. In a later study (Alary et al. 2009), the authors aimed to generalise this finding to other discrimination tasks and reported that the blind participants' advantage was not task independent. In this study, there were no differences between the groups (composed by the same participants who collaborated in the earlier study) in haptic perception for a grating orientation task and for a vibrotactile frequency task. However, blind participants performed better in a discrimination of texture task, which the authors attributed to the possible similarity between the presented dotted textures and Braille characters.

Blind participants have shown faster performance in shape correspondence task (Postma et al. 2007). In this study, in a speeded task in which participants were asked to match shapes to their negative in a board as fast as possible, the blind participants' group was faster, but not more accurate than the sighted participants' group. However, in a non-speeded task, no differences were revealed between the two groups.

In a study on haptic concepts (Homa et al. 2009), four groups (blind participants, sighted blindfolded participants, sighted and touching participants and sighted only participants) were asked to perform classification and recognition tasks with a set of objects varying on texture, shape and size. Results revealed that all participants took approximately the same amount of time to learn the categories. However, blind participants differed from the other groups by presenting fewer false alarms to new patterns that belonged to the category, and revealing more false alarms to the category prototype, which was falsely recalled in all trials.

Stevens et al. (1996) showed that tactile acuity decreased as a function of age at the same rhythm for sighted and blind participants, resulting in slower Braille reading in the blind participants' group.

Equivalence from spatial processing in vision and touch has also been reported in research with blind participants. For instance, learning of maps through vision in sighted participants or through touch in blind participants showed 
similar results, suggesting that haptic information does not need to be translated into visual codes and had direct access to spatial processing (Giudice et al. 2011).

Pring and Rusted (1985) showed that blind children could benefit as much from haptic pictures as sighted children benefit of visual pictures in educational settings. Children either heard a description of a rare animal or haptically explored a picture of the animal, and results showed that children in the second condition were able to remember more information about the animals.

Another interesting and large group of results reported the existence of visual imagery in blind participants, questioning the notion that imagery is visual in nature, and suggesting the existence of shared components between vision and haptics (e.g., Zimler and Keenan 1983; and for a review Cattaneo et al. 2008). According to some authors, mental imagery has to be understood as a group of complex processes that generate representations, and not only as a perceptual trace. From this point of view, mental images do not need to be visual in nature and can be elicited by other sensory modalities (e.g., Cornoldi et al. 1998).

The previously presented studies allow the clarification of some concepts and permit a better understanding of how the brain works and adjusts to sensory deprivation. Studies with blind subjects make it possible to conceive a scheme of the brain where perception is not dominated by vision, and in which other sensory modality processes can be explained without the need to hypothesise the conversion of information to visual codes, even when we are talking about mental images.

Overall, some studies with blind participants have suggested advantages in haptic perception when compared to sighted participants (e.g., Heller and Ballesteros 2006). Nonetheless, the role of experience and familiarity has to be considered in data interpretation and performance of blind and sighted subjects in conditions that are novel or highly familiar for both groups, as well as conditions that are highly familiar to both, seems to be largely equivalent.

\section{Children}

Developmental approach to touch is interesting to understand how human perception evolves through life span and can also provide some insight into how information acquired by touch is processed and weighted at different ages.

Studies with very young children (before the development of language) are relevant because they allow access to truly perceptual tactile features, since young children are not capable of semantically or verbally encode or rehearse the stimuli (Gallace and Spence 2009). There are some studies regarding touch in newborns and infants, mainly recurring to the novelty paradigm. This paradigm is based on the conclusion that babies tend to be attracted by new stimuli and was designed on the assumption that the time a child spends exploring a stimulus provides a measure of its novelty. Longer exploration times indicate the stimulus is being perceived as new. With the novelty paradigm, the existence of some form of tactile memory has been reported in children as young as two months old (Lhote and Streri 1998), and even newborns were capable of detecting changes in two stimuli with either hand (Streri et al. 2000). Also, Catherwood (1993), in a study with infants with about eight months of age, showed retention of tactile information for both shape and texture with immediate recognition, for shape and to lesser extent texture after a delay and only for texture in interference conditions.

Corbetta and Snapp-Childs (2009) highlighted the role of touch in early development, considering that coordination between touch and vision is crucial to maintain an interaction with the surrounding stimuli, and trying to analyse the relative weight of tactile and visual experience in the modulation of grasping, reaching and other objectoriented motor responses of six- to nine-month-old children. Results showed that younger children did not adapt their movements according to previous experience with the stimuli, but older children did, relying first on touch information and later more in visual cues to match their movements to the specific objects, based on previous experience with them (Corbetta and Snapp-Childs 2009).

Bigelow (1981) reported a study with two groups of children, one with children around two and a half years old and the other with children of 5 years old and asked them to haptically identify miniatures of small objects (e.g., doll's spoon), miniatures of large objects (e.g., doll's sofa) and small objects in real size (e.g., key). Older children were better than younger children in all conditions, and both groups were better at identifying small objects in real size than either of the two other object sets. Curiously, the younger children were able to identify more small miniaturised objects than large miniaturised objects, a pattern that was not observed in older children. The author concluded that younger children had more difficulty in haptically perceiving and exploring objects they cannot compare in real size, and the greater difficulty in identifying the miniatures of large objects was a consequence of lack of experience of haptic exploration of the objects in real life. As for the older children, their crossmodal abilities were more developed, and they were able to identify the objects by translating the visual information from real life to haptic information. Bushnell and Baxt (1999) in a recognition study with familiar (e.g., toothbrush) and unfamiliar (e.g., feather boa) objects with a group of 5-year-old children reported a "remarkably good" haptic memory, nearly perfect for familiar objects and excellent for unfamiliar objects as well. 
The exploratory procedures of children up to 11 years old did not seem to be strictly object- or property-dependent (Hatwell 2003), with children performing the same movements, independently of the given instruction, while adults adjusted the exploratory procedures both to object and to task specificity. Haptic perception relies highly on movements and intentional exploration, and as such is dependent on the development of the motor system; likewise, the development of cognitive abilities like working memory is important to adjust, maintain and bind acquired information that allows haptic perception and motor optimisation of the manipulation procedures (Hatwell 2003).

Children show the ability to retain haptic specific information from birth and seem to initially use mainly motor and haptic features to guide objects' exploration. However, the accuracy in haptic recognition of unfamiliar items and the ability to adjust exploration procedures seem to develop later.

\section{Sex differences}

Literature on touch and sex differences appears mainly in social and interpersonal research, analysing touch as a form of communication (e.g., Gallace and Spence 2010; Jones 1986; Stier and Hall 1984; Willis and Hofmann 1975). However, other studies have explored sex differences in touch within experimental tasks.

Bardwick (1971) presented a review of studies about tactile perception in females and concludes that the evidence for sex differences was scarce and incongruent, and for that reason argued in favour of assuming no differences in performance. Researchers have discarded sex differences analysis from their works, and research on haptic perception has focused in establishing general rules of processing, regardless of the participants' sex. However, some studies can be identified in which sex differences are reported.

Heller et al. (2010) explored differences between women and men in a study in which participants had to detected changes of position in raised line drawings presented in a matrix. Results revealed that female participants presented better results than male participants, but these differences disappeared in more difficult tasks. Overall, the reported sex difference was small and congruent with the differences found in vision for a similar task.

Analysing the perception of tactile simultaneity of pairs of stimuli did not result in consistent sex differences between participants, reporting that male performance was faster, but not in all conditions (Geffen et al. 2000a, b).

Using positron emission topography, Sadato et al. (2000) described differences between men and women in the activation of the dorsal premotor cortex, which was asymmetrical in men but symmetrical in women. The authors concluded that this might suggest differences between the groups in tasks such as discrimination through active touch, where inter-hemispheric connection would be stronger in women, facilitating performance.

In haptic orientation perception, differences were found between male and female participants (Zuidhoek et al. 2007). In this study, participants were distributed through three experiments, in which they were asked to touch a fixed plastic bar with one hand and orient a movable plastic bar with the other hand in a way that both bars were parallel (Experiment 1); use one hand and, considering the bar as a pointer in a clock, provide a verbal answer identifying the represented minutes for each orientation (Experiment 2 ); orient a bar as a clock pointer according to the verbal indication of the experimenter (Experiment 3). Results showed that males were more accurate in parallel setting and haptic orientation perception.

Although there are some studies reporting differences between male and female participants in some specific haptic tasks, this variable has not been part of most systematic studies on touch. In addition, experimental results of sex differences manipulations are not very clear, usually presenting small or inconsistent effects.

The study of touch according to the participants' group can add relevant knowledge. Studies with blind participants allow the evaluation of touch performance in individuals with a high degree of specialisation and experience in tactual interaction of stimuli, and a clear analysis of tactual performance without visual mediation that is not available in blindfolded sighted participants. Experiments with children and infants can bring an interesting development perspective to how we touch and how we perceived touched stimuli and can even allow the evaluation of tactual perception independently of verbal mediation (with preverbal infants). Finally, the summary of research directly addressing sex differences in touch revealed that this dimension is still unexplored and that the few studies that address it do not seem to imply a systematic effect of sex in tactual perception.

\section{Type of stimuli exploration}

The next section of experiments is organised attending to the stimuli exploration procedures. First, the differences between passive and active touch are addressed, then we will focus in the relevance of movement in tactual perception and finally, we will consider the exchange between the tactual manipulation and time in the presented tasks.

Passive and active touch

In touch research, three main types of touch are distinguished: passive touch, active touch and dynamic touch. 
Passive touch refers to conditions in which participants are not allowed to move, and the stimuli are presented against the skin. This type of touch is used with air jets (e.g., Bliss et al. 1966), vibration (e.g., Gallace et al. 2008), or even by pressing still stimuli against the participant's skin (e.g., Cronin 1977). Active touch implies movement from the participants and can be: 1) free, when the participants are allowed to explore the objects with the whole hand (e.g., Klatzky et al. 1985); or 2) restricted, when participants can touch the objects: with only one finger (e.g., Klatzky and Lederman 1995), through gloves (e.g., Klatzky et al. 1993), or with a specific type of movement (e.g., Lederman and Klatzky 2004). Gibson (1962), in an analysis of active touch, emphasised the exploratory nature of active touch, in contrast to the receptive nature of passive touch, which is caused by an external agent, and additionally argued that active touch was not equivalent to a simple adding of passive touch and kinaesthesia. Dynamic touch was defined by Gibson (1966) as the perception that results from the combination of cutaneous, muscular and joints cues. In such conditions, the stimuli are moved through the participant's skin, allowing the perception of movement cues not present in passive touch, but not requiring the voluntary exploration present in active touch (e.g., Turvey 1996; Sanders and Kappers 2008). Experimentally, dynamic touch is a condition that allows higher experimental control than active touch, since participants are not allowed to move their hands, minimising variations in exploration. This type of touch is sometimes referred to as an option of passive touch, in which the stimuli are moved by the experimenter (e.g., Cronin 1977) or by a mechanical device against the participant's skin allowing the participant to perceive relevant cues that result from movement, without having to actively manipulate the stimuli.

Another author analysed the differences between active and passive touch in the identification of geometrical forms (Cronin 1977). Comparing participants in the first, third and fifth grade and college students, the author concluded that a developmental advantage existed for active touch (the participants actively touch the object) and dynamic touch (the stimulus is moved against the participant's hand) conditions, but not for passive touch conditions, in which 5-year participants showed differences compared to the college students, but not compared to third- and fifth-year students. The author argued that the equivalence in performance between active and dynamic touch might be explained by the fact that participants had to explore the objects with the palm of the hand, and not the finger, which could have limited the type of information gathered in active touch conditions, making it similar to dynamic touch. Overall, conditions that involved movement resulted in better performance than passive touch, in every age group.
In a study comparing the perception of forms in active and passive exploration conditions (Heller 1984), results also pointed to a clear advantage of active touch, with passive static or sequential presentation resulting in worst identification than active touch.

In some conditions, passive touch might present an advantage when compared to active touch. For instance, in a recent study comparing tactile learning of a maze (Richardson et al. 2011), participants in the passive touch condition were much faster to learn the path than participants in the active touch condition. Nonetheless, the disadvantage in the active conditions was due to the repetition of errors in the chosen paths and was a consequence of the participants' decisions. As such, the authors concluded that the active touch disadvantage translated into a cognitive limitation but not a haptic system limitation (Richardson et al. 2011). There can be conditions where active touch shows no advantage in performance. Guclu and Murat (2007) published a study comparing active and passive touch in a counting task. Participants had to count the number of bumps presented in sticks that were either actively explored or slid against the participants' hands. Results showed that active touch did not imply a better performance.

Active touch and passive touch are distinct processes. While active touch involves proprioception, kinaesthesia and cutaneous senses, passive touch relies only on the latter. Interestingly, electrophysiology studies have reported that active touch generates a suppression of afferent information to the somatosensory cortex, a phenomenon known as movement-related sensory gating (e.g., Chapman 1994). This phenomenon could lead to worst encoding in active than passive touch. Nonetheless, active touch allows the participant to control movement velocity and to select which properties to evaluate at each moment, resulting in a central advantage for active touch (Chapman 1994).

Using functional Magnetic Resonance Image (fMRI) in a study that required evaluation of roughness in active (participants moved their fingers through the samples) and passive (the samples were moved beneath the participants' fingers) touch, it was possible to verify that exploratory conditions affected activation in the primary sensory cortex and that active touch resulted in a larger pattern of activation, possibly because of the motor component of this condition (Simões-Franklin et al. 2011).

Although considering some exceptions to the active touch superiority, it seems that this type of stimuli exploration in touch is ideal to observe optimal performance. Although passive touch allows a great control of manipulation variables, active touch can be studied and explored with controlled experimental paradigms and seems to be able to provide more information about processing in touch (Gibson 1962). 
Relevance of movement in touch

The relevance of movement in touch was valued since the first efforts to systematically study this modality. Gibson (1966), Katz (1925/1989) and Weber (1834/1996) and 1905/1996) understood the relevance of dynamic contact with the stimuli from their early experiments and considered the sense of movement crucial in tactile perception. Later, the works of Lederman and Klatzky (e.g., Lederman and Klatzky 1996) brought a first effort to give some structure to our knowledge about movement in touch. Nonetheless, despite the importance of movement to touch, most studies with tactile stimuli are still conducted with passive stimulation (e.g., Spence and Gallace 2007). In fact, the variability of hand movements that people perform to explore an object presented by touch only makes it harder to control for exploration times and inter-stimulus intervals and introduces some individual variability in the way stimuli are explored, even though general movement patterns are always detectable (Lederman and Klatzky 1987).

Object manipulation is determinant for the acquisition of object properties like texture, shape, weight or volume. The movements performed while touching an object are pertinent to the perception of the object in space (Kaas et al. 2008), and grasping has been shown to be a crucial stage in object identification as well (Klatzky 1992), showing the relevance of active manipulation for touch.

Lederman and Klatzky (1987) provided a systematic classification of manual object exploration. In this study, participants were asked to touch everyday objects, without sight, and to evaluate specific features like weight. Participants were allowed to explore the objects freely with both hands. Through a complex movement analysis that involved video examinations by naive evaluators, the authors were able to discriminate between six essential movement patterns, each one specified in collecting particular information from the objects: lateral motion (texture), pressure (hardness), static contact (temperature), unsupported holding (weight), enclosure (volume, global shape) and contour following (exact shape). The authors called these movement patterns as exploratory procedures and defined them as a stereotyped movement pattern having certain characteristics that are variant and others that are highly typical (Lederman and Klatzky 1987, p. 344). By this definition, the authors explained that it is possible to identify general patterns of movement, equivalent in all participants (for instance, rubbing an object to evaluate its texture), although there are some variations in the individual exploration (for instance, some participants rub the object with only one finger while other might use two fingers or even the whole hand).

More recently, using a movement-tracking device, Kappers and colleagues (Bergmann-Tiest et al. 2010;
Kappers and Douw 2010) initiated research on the relevance of movements while exploring two-dimensional stimuli. Bergmann-Tiest et al. (2010) were able to extract specific patterns of exploration from the movement coordinates recorded through the experiment and were able to establish relations between these patterns and specific properties' evaluations, namely hardness, coldness, texture and texture orientation. Kappers and Douw (2010) work showed that participants performed the same type of movements when trying to answer to the same questions exploring a stimuli matrix (e.g., Which sample is the warmest?). These studies showed that haptic movements are specific and directed to feature evaluation and that the movement patterns noted by Lederman and Klatzky (1987) regarding three-dimensional everyday objects also emerge with two-dimensional stimuli, although the movements that characterise each pattern might suffer some alteration due to stimuli specificity (e.g., global shape and shape contours correspond to the same procedure for two-dimensional objects, because participants cannot hold these type of stimuli).

\section{Exploratory movements and time}

Haptic processing is sequential (Loomis et al. 1991) implying a progressive apprehension of discrete stimulus properties, contrasting to the simultaneous perception of the whole stimulus that occurs in vision. The consequence of this type of processing is that haptic exploration takes longer than vision. Vision, on the other hand, represents parallel processing, that is, being able to collect simultaneously a set of different properties from the stimuli. In an experiment designed to compare visual and haptic processing in sequential processing, Loomis et al. (1991) restricted the visual field of view in object exploration, allowing the participants to look sequentially to a small area in different points of a stimulus. Within these conditions, performance was very similar in haptic and visual perception, revealing that the type of processing significantly affected the participants' ability to recognise stimuli. This distinction is crucial, and when planning haptic experiments, one should allow sufficient time for haptic stimuli exploration.

In a review on sensory modality effects, Freides (1974) noted the differences in time resolution between sensory modalities. Goodnow (1971) observed that when delays increased, shape-matching in touch was much more affected than in vision. Also, Wagner and Sakovits (1986) indicated that vision was capable of processing more information than touch within the same time.

Lacey and Campbell (2006) reported that the ratio in presentation times has to be of 2:1 when comparing haptic to visual presentation, a conclusion they came across 
during a set of pilot experiments. Woods et al. (2004) described that matching performance levels in a crossmodal paradigm with vision and touch implied that stimuli presentation in touch took twice the time of the visual presentation. Likewise, in a study with three-dimensional complex objects, built with toy construction blocks, it was found that visual presentation required half the time as haptic presentation (Newell et al. 2001).

Time is a crucial variable in perception and memory, and our ability to maintain a certain stimulus in memory actually contributes to the definition of that specific memory storage. For instance, a partial report paradigm in a numerosity task with tactile stimulation showed that in short-term registers, participants can access information that they could not report in a regular tasks requiring a complete recall (Auvray et al. 2011). As such, in tactual experiments, if the participants are taking longer to perceive the stimuli, they will take longer to perform the task, which can result in a need to establish specific durations of haptic memory stores. Furthermore, the increased presentation times in haptics make comparison with vision and audition very difficult in traditional memory paradigms, such as memory span or even n-back tasks. A direct comparison between performances in all sensory modalities in similar tasks might not be possible, but it is necessary to explore haptic memory attending to its specificity and perhaps comparing haptic performance in optimal haptic conditions in contrast to conditions in which other modalities have already been evaluated (for instance, with shorter time intervals). In this context, analysing visual and auditory tasks with equivalent times to haptic optimal performance might enlighten how haptic information is processed and clarify if haptic memory storages present longer durations than visual or auditory stores.

As was mentioned throughout this section, movement cues are essential for touch and they allow the extraction of unique features of the stimuli, determining participants' performance. Enabling an active interaction with the stimuli besides presenting a more natural exploration also implies a higher degree of feature perception, optimising tactual perception. Even though usually a free exploration condition implies a longer interaction with the stimuli and a longer interval between study and test, and the time factor is very relevant in perceptual but specially memory tasks and as such must be considered.

\section{Touch and the other senses}

Our everyday interaction with objects is mainly multisensory. We are able simultaneously to perceive the colour of a fruit, its fragrance, softness, the sound of the bite and its sweetness, and all of these different perceptions seem to be deeply integrated to the point that we are able to recall an apple's taste and smell just by seeing its picture.

The interactions between the senses are crucial to the knowledge of cognitive processes. The ability to share stimulus properties between senses and at the same time, the specificity that each sensory modality brings to our everyday experience are relevant topics in human cognition. Possibly due to the complex relations, interactions and distinctive features among sensory modalities, the topic of multisensory and crossmodal perception is not yet developed more in higher order processes such as memory. Nevertheless, the topic of crossmodal interactions has interested researchers through history and has shown that the combination of information from different sensory modalities is useful allowing the correction of biases generated by a particular modality. As an example, having a variety of sources to perceive the same object or event allows us to reduce perceptual ambiguity (Helbig and Ernst 2008) and produce more efficient responses. Through this section, we will analyse research that connects touch to vision, audition, taste and smell.

\section{Touch and vision}

Haptic research has been mostly conducted under multimodal visuo-tactile paradigms, either comparing performance on touch and vision (Bergmann-Tiest and Kappers 2007; James and Blake 2004; Kappers and Schakel 2011) or exploring shared representations between the two modalities (Easton et al. 1997a, b; Lacey et al. 2007; Whitaker et al. 2008).

Lacey et al. (2007) conducted a review on vision and touch interactions attending to crossmodal memory studies from a theoretical perspective. The authors argued that, in memory tasks, participants have to retrieve a representation of the object, and as such these tasks would be ideal to test the nature of crossmodal representations. The authors concluded that there is enough evidence to assume that both modalities share cognitive resources and processes, as shown by processes such as viewpoint dependence on vision and touch and the equivalence of spatial representations in the two modalities. These representations were described as dynamic and could be affected by both topdown and bottom-up processes. Even though the authors highlighted the relevance of research that has reported differences between the two modalities and has pointed to the existence of unimodal coding and retrieval processes, for instance, better performance in memory tasks when the presentation modality is the same as the retrieval modality. As such, they concluded that further research was needed to clarify these modality-specific processes (Lacey et al. 2007). These descriptions of shared generic components, marked by modality specificity, are the most frequent 
report in crossmodal visuo-tactile studies, as will be illustrated in this section.

A central aspect of the connection between vision and touch is the shared spatial component, present in both modalities, but mostly explored in vision. It seems that there is an equivalence between touch and vision for spatial processing, and many researchers have explored this topic. Easton et al. (1997a, b) present a study consisting in the presentation of words either visually in a computer screen or haptically through printed raised letters. Participants previously studied 10 words visually and 10 words haptically and at test had to perform either a word-stem task or a cued-recall task with 30 words (20 studied and 10 nonstudied) in one of the modalities. The authors reported no differences between modalities and argued in favour of shared representations in vision and touch. In another study, Easton et al. (1997a), aware of the relevance of verbal processing in the presentation of words, explored visual and haptic representations in a similar paradigm, but testing participants with two-dimensional patterns and three-dimensional objects. The participants had to study part of the stimuli visually or haptically, and then their task was to either perceptually identify item or explicitly recognise it. Results showed that for two-dimensional patterns, cross-modality priming was strong (meaning that visually studied objects were haptically identified or recognised better than non-studied objects and vice-versa). With three-dimensional objects, modality-specificity was observed indicating that both shared and unique processes determined crossmodal integration.

In a study with blind and sighted participants, Giudice et al. (2011) asked participants to learn four-point routes in a map either visually or haptically and then to reproduce the path in the test phase. Results showed that spatial images were shared between vision and touch. Results for the blind participants' group suggested that this equivalence was not a consequence of visual recoding of haptic information, since the pattern was equivalent for both participants' groups. Likewise, Cattaneo and Vecchi (2008) asked participants to memorise a number of locations in a $5 \times 5$ matrix either visually or haptically and to report them in an empty matrix. Results showed that visual performance was overall better than haptic performance, that representations were modality-dependent and that some information was shared between modalities, indicating that both supra-modal and modality-specificity processes were present.

Besides spatial equivalence, other similarities have been reported for visual and haptic processing. Luo and Imamiya (2003, 2004) reported that colours affected perceived haptic roughness in surfaces. In texture perception, Picard (2006) reported partial equivalence between vision and touch in a crossmodal matching task, in which participants had to match stimuli that were previously rated as presenting a high- or low-crossmodal dissimilarity. This study broadened the findings of Garbin (1988) on visuo-haptic equivalence for shape perception. A review on texture perception by vision and touch (Whitaker et al. 2008) concluded that, unlike shape perception, texture perception by vision and touch was not equivalent, but complementary. The authors pointed to research providing no evidence for visuo-haptic integration in behavioural tasks with familiar objects (e.g., Bergmann-Tiest and Kappers 2007) and suggested that this lack of crossmodal correspondence might be due to more elaborate cognitive processes than basic perception.

Crossmodal illusory conjunctions (considering that an item presented in one modality was actually presented in another) were also observed within touch and vision (Cinel et al. 2002). Participants saw visual stimuli while touching tactile stimuli and after a mask presentation had to report the shape and texture of the seen object or the orientation of the touched stimuli. Results showed that participants reported felt textures as seen and vice-versa, and these misguided reports were more frequent when visual and haptic stimuli were presented in the same hemispace. Auvray et al. (2007) also described equivalence between touch and vision in a crossmodal change blindness paradigm. Change blindness is a phenomenon characterised by the inability to detect differences in two consecutive stimuli, due to the presentation of a mask between them. This phenomenon has been studied in vision, audition and touch (Auvray et al. 2007). In this study, change blindness was reported even when stimuli were presented in different modalities (haptic and visual), and regardless of the mask presentation modality, although the phenomenon was stronger in within-modality presentations. According to the authors, these results indicate that multisensory processes could explain change blindness.

Craddock and Lawson (2009a) argued that size perception was shared between vision and haptics after a crossmodal study in which participants were asked to recognise three-dimensional objects based on shape alone, ignoring size changes. Results revealed that size variation impaired crossmodal recognition, suggesting shared representations of size between modalities. The study did not allow a clarification on the nature of these shared representations, and the authors hypothesised that they might be either perceptual (low-level) or mediated by high-level processes.

Another group of studies has focused on the differences between touch and vision in perception and recognition tasks. Lawson (2009) evaluated depth rotation in visual and haptic conditions with morphs of everyday objects in a sequential-matching task in within modal and crossmodal conditions. The difficulty of the task was manipulated 
through the discriminability of the presented stimuli (from presenting unrelated stimuli-bed, lizard-to presenting related stimuli-bed, chair-and finally presenting very similar stimuli-bed, bed-chair morph). Results showed that for within modal and visuo-haptic trials, object discriminability and orientation changes impaired recognition, while in haptic-visual trials orientation changes had no effect. Also, orientation changes and discriminability had additive effects for visuo-haptic trials, but haptic-visual trials did not reveal orientation change effects. This latter result suggests that different processes underlie haptic and visual orientation processing.

In a study analysing the perception of shape and size by vision and touch through a computer screen and a Phantom system, Van Doorn et al. (2010) asked participants to evaluate the size of touched shapes (squares and lines), selecting the touched shape from a haptic or visual set. Haptic exploration was either active (participants actively moved the Phantom) or passive (guided by Phantom) and could present cutaneous information (direct contact with a raised line) along with kinaesthetic information (movement through the stimulus). Performance in the visual task was better than in the haptic task when participants were allowed to passively explore the objects, but when cutaneous information was allowed in active exploration, haptic performance was better than visual.

In another study, examination of visual and haptic perception of shape for three-dimensional objects resulted in a partial functional equivalence between modalities, with noticeable unimodal representations (Norman et al. 2004). In this experiment, participants were able to recognise objects in crossmodal conditions with high levels of accuracy; nonetheless, performance was better in unimodal conditions. Stadtlander et al. (1998) explored visual and haptic recognition in function of age and concluded that regardless of sensory modality, young adults (20-35 years old) were better than older adults (60-75 years old) in object recognition. Also, haptic recognition supported the higher recall in both groups (younger and older participants).

Ernst and Banks (2002) developed a multisensory integration mathematical model applicable to vision and touch interactions, as well as other multimodal associations (e.g., Bresciani and Ernst 2007). This model is based on Bayesian statistics and uses maximum likelihood estimation, assuming that visual and tactile inputs are integrated in a statistically optimal fashion (Ernst and Banks 2002). According to this model, when two perceptual modalities are capable of processing the same stimuli, the most influential modality will be the one that presents the lesser variance in perceptual estimation. This model allows a better understanding of apparently contradictory experimental findings in which vision dominates for some tasks and haptics for others. According to the maximum likelihood model, the dominant modality will be the one with more reliable information for each particular task.

Previous studies focused essentially on the analysis of visuo-haptic interactions for stimuli features that can be encoded by both modalities: location, patterns, texture, shape, size and orientation. For these common features, partially shared processing is described in the literature, with many features presenting equivalent manipulation and representation in both modalities (e.g., location), but others maintaining modality specificity (e.g., texture).

Touch and audition

Audiotactile interactions have been reported in two main domains: temporal and spatial. The temporal domain is related to a large group of research evaluating the perception of temporal order in tactile and auditory stimuli when presented with different time intervals (e.g., Occelli et al. 2008). The spatial domain focuses on the perceived simultaneity of tactile and auditory stimuli presented at a same location in space and concentrates on the apparently automatic integration of this type of information (e.g., Zampini et al. 2005).

It has been shown that beep sounds can modulate the counting of tactile taps (Bresciani et al. 2005). Participants were asked to concentrate in the number of taps and to ignore the beeps; nonetheless, numerosity judgements were biased by simultaneous beep presentation. As the interval between tap and beep was increased, the modulation effect decreased, suggesting that simultaneously presented audiotactile information tends to be integrated (Bresciani et al. 2005). In a later study with similar procedure, the authors concluded that not only auditory signals affected tactile processing, but also tactile taps affected auditory counting and reported that diminishing the intensity of sounds generated less interference on tactile counting, as well as affected the impact of touch on the auditory task (Bresciani and Ernst 2007). As such, not only audiotactile integration was shown to be automatic, but also it was revealed to be signal-reliability dependent.

Gillmeister and Eimer (2007) reported tactile enhancement of auditory perception with two experiments evaluating detectability and intensity of auditory stimuli. In the first experiment, participants had to signal if an auditory stimulus was presented in one of two time intervals, defined by a visual mark. Results showed that tactile stimuli facilitated the detection of sound and that this effect was larger on simultaneous trials (in which tactile and auditory stimuli were presented at the same time) than on asynchronous trials (in which tactile and auditory stimuli were presented with a time interval between them). The second experiment implied a stimuli intensity task, consisted in the evaluation, in a nine-point scale, of tactile and 
auditory stimuli. Tactile stimulation led to higher intensity judgements of the auditory signal. Again, simultaneous presentation resulted in better integration between modalities, revealing that temporal synchrony is an essential condition for audiotactile integration.

Interactions between audition and touch were found to be dependent on hand position in a study reported by Sanabria et al. (2005a). Participants were asked to place their hands either crossed or over the midline and had to discriminate the direction of an auditory array (sequence of sounds presented in different spatial locations) while receiving spatiotemporal congruent or incongruent tactile stimulation in the hands. Impaired performance was reported for incongruent tactile stimulation, but only for participants who did not have crossed hands. In a second experiment, the authors used the same procedure but asked participants to focus on the tactile stream and not the auditory one and found that the interaction effect was stronger in the crossed hands condition. The authors concluded that body posture could affect crossmodal interaction, and audiotactile influences seemed to be reciprocal (Sanabria et al. 2005a).

In an exploration of audiotactile integration in blind and sighted participants, Hötting and Röder (2004) asked participants to estimate the number of rapidly presented tactile stimuli while ignoring non-relevant sounds during the task. When a tactile stimulus was presented in conjunction with more than one sound, participants reported feeling more than one touch, and this effect was stronger for sighted than blind participants.

Crossmodal perception between touch and audition has also been tested with other paradigms like the saltation illusion, when one target stimulus is systematically displaced in the direction of another stimulus that is presented either at a near position or at the same time (e.g., Trojan et al. 2009); the ventriloquist effect, when stimuli from two sensory modalities presented from a same spatial location are attributed to the same source (e.g., Bruns and Röder 2010); and apparent motion phenomenon, when a sequence of presented stimuli is perceived as one stimulus in motion (e.g., Sanabria et al. 2005b). In all these paradigms, the presence of an automatic integration of auditory and tactile stimuli is reported, and the effect seems to be stronger when both stimuli are presented simultaneously, especially when the stimuli are presented in the same spatial location, indicating a natural form of integrated perception between modalities.

Touch, smell and taste

Olfaction and taste are research topics that have been studied mainly in the food industry, and within this context, research on touch has been essentially restricted to the relevance of perceived textures and temperature in the tongue while tasting food.

The connections between touch, smell and taste were already mentioned in the literature many years ago. Gibson (1966) suggested the definition of flavour as a specific sense that involved inputs from taste, smell and touch. Titchener (1909) also mentioned the relevance of a combined perception of temperature, touch, taste and smell in the ingestion of coffee or fruit.

A review on the multisensory perception of flavour (Delwiche 2004) enhanced the role of touch influencing perceived flavour through temperature and texture perception. The interaction between touch and taste was clear in a study that revealed a tactile-taste illusion caused by thermal stimulation (Cruz and Green 2000). In this illusion, after putting an ice cube on the side of the tongue, a salty taste was perceived, originating a taste perception after temperature stimulation. Besides the strong salty sensation, the authors were also able to induce perceptions of sweet (by warming the anterior edge of the tongue after a cold temperature), sour and bitter (by cooling the tongue). Another reported interaction between touch and taste was that the perceived viscosity of a liquid can alter the intensity of its flavour to the taster (Bult et al. 2007). In a more recent review on this topic, Auvray and Spence (2008) also argued in favour of a multisensory perception of flavour, derived from taste, smell and tactile cues, which continuously affect each other when we are eating.

The connections between touch and smell are also explored in the literature. Laird (1932) conducted a study in which women judged the quality of silk stockings. Without the knowledge of the participants, the stockings were impregnated with light fragrances, and women consistently evaluated identical stockings as being better when they presented a narcissus fragrance, and justified their evaluations with properties as durability or sheen, but never attending to olfactory characteristics. The presence of odours can also affect the evaluation of fabric softness, as was shown in a study in which fabrics associated with lemon odours were evaluated as softer than fabrics associated with animal odours (Demattè et al. 2006).

The understanding of the apparently automatic processes that allow interchange of information between sensory modalities, enabling reciprocal modulation in ongoing tasks, is crucial in cognitive sciences. The need to control for any multisensory interactions when trying to access processes in a specific sensory modality, as well as the exploration of these natural connections between the senses in memory processes, is clear. The nature of sensory representations, either in their suggested amodal or higher order processing, or concerning intra-modal particularities needs to be acknowledged in order to strengthen experimental designs and procedures. 


\section{Conclusion}

This review aimed to classify previous studies on touch according to their procedures and experimental conditions, systematically compiling the previous literature and its empirical and theoretical implications, presenting a state of the art for tactual research.

We evaluated the way that different features affect results in touch experiments and considered the consequences of these manipulations for empirical studies. As such, we analysed: interactions between touch and other senses; the impact of the type of stimuli (concrete and abstract); the type of participants; and the type of stimulus exploration that is adopted. A systematic assessment of the effects of these dimensions was not available in the literature, and it provides both a general introduction to all the specific features that one must consider when designing experiments on touch and also an overview of the main results and theoretical implications, driven from the previous literature.

In a first approach, performance in touch experiments seems to be highly variable, and it is important to focus on each condition that might be contributing to those seemingly incongruent results in haptic performance. Further knowledge on touch has to consider all the possible influences in touch performance.

The conditions, in which touch is evaluated, determine our understanding of this sensory modality and limit the interpretation and possible generalisation of results. The target stimuli of an experiment (patterns, familiar objects, uncommon shapes, complex matrices, etc.), the presentation conditions (serial or parallel, through active or passive touch) as well as the chosen participant sample (children, younger adults, older adults, healthy participants, mild cognitive impaired participants, blind participants, etc.) should be attended and considered when trying to generalise research results and draw implications for theoretical perspectives in human cognition.

The nature of the presented stimuli, the understanding of the specificities of the participants (e.g., age) in the experiment and the conditions in which manual exploration is allowed are central variables in the study of touch. All of these dimensions contribute to the understanding of differences and similarities between touch and other sensory modalities, as well as to the exploration of the existence of amodal or modality independent processing modes that allow exchange of information between sensory modalities.

Besides the experimental implications and the clearer understanding of how each experimental manipulation might affect the results of tactual studies, this review presented a summary of the main findings in tactual research. All the reported dimensions in tactual research contributed to a better and larger knowledge about the cognitive processes underlying touch. Research on touch is becoming more frequent and specialised, and this interest in how we perceive, process, elaborate and recall touched items is allowing a fast development of the area in the later years. As we have seen, previous research has shown that touch is not an inferior or ineffective sensory modality. The adaptation of experimental procedures and the effort to test tactual performance in optimal conditions have contributed to the definition of touch as a highly efficient and reliable modality, and this knowledge can be crucial to the development of tactual enhanced systems in applied areas, whether considering sensory substitution, human-computer interactions, warning systems, emotional design, or others.

Acknowledgments This work was funded by the Portuguese "Foundation for Science and Technology" through $\mathrm{PhD}$ scholarship SFRH/BD/35918/2007.

\section{References}

Abramowicz A, Klatzky RL, Lederman SJ (2010) Learning and generalization in haptic classification of 2-D raised-line drawings of facial expressions of emotion by sighted and adventitiously blind observers. Perception 39(9):1261-1275. doi:10.1068/p6686

Alary F, Goldstein R, Duquette M, Chapman CE, Voss P, Lepore F (2008) Tactile acuity in the blind: a psychophysical study using a two-dimensional angle discrimination task. Exp Brain Res 187(4):587-594. doi:10.1007/s00221-008-1327-7

Alary F, Duquette M, Goldstein R, Chapman CE, Voss P, La Buissonnière-Ariza $V$, Lepore $F(2009)$ Tactile acuity in the blind: a closer look reveals superiority over the sighted in some but not all cutaneous tasks. Neuropsychologia 47(10):2037-2043. doi: 10.1016/j.neuropsychologia.2009.03.014

Auvray M, Spence C (2008) The multisensory perception of flavor. Conscious Cognit 17(3):1016-1031. doi:10.1016/j.concog.2007. 06.005

Auvray M, Gallace A, Tan HZ, Spence C (2007) Crossmodal change blindness between vision and touch. Acta Psychol (Amst) 126(2):79-97. doi:10.1016/j.actpsy.2006.10.005

Auvray M, Gallace A, Spence C (2011) Tactile short-term memory for stimuli presented on the fingertips and across the rest of the body surface. Atten Percept Psychophys 73(4):1227-1241

Bach-y-Rita P (1972) Brain mechanisms in sensory substitution. Academic Press, New York

Ballesteros S, Manga D, Reales JM (1997) Haptic discrimination of bilateral symmetry in 2-dimensional and 3-dimensional unfamiliar displays. Percept Psychophys 59(1):37-50

Bancroft T, Servos P (2011) Distractor frequency influences performance in vibrotactile working memory. Exp Brain Res 208(4):529-532. doi:10.1007/s00221-010-2501-2

Bardwick J (1971) Psychology of women. Harper \& Row, Michigan

Bergmann-Tiest WMB, Kappers AML (2007) Haptic and visual perception of roughness. Acta Psychol (Amst) 124(2):177-189. doi:10.1016/j.actpsy.2006.03.002

Bergmann-Tiest WWB, Norman J, Kahrimanovic M, Kappers AML (2010) Hand movements during haptic object exploration. Perception 39. ECVP abstract supplement, p 132

Bigelow A (1981) Children's tactile identification of miniaturized common objects. Dev Psychol 17(1):111-114

Bliss J, Crane H, Mansfield P (1966) Information available in brief tactile presentations. Percept Psychophys 1:273-283 
Bresciani J-P, Ernst MO (2007) Signal reliability modulates auditorytactile integration for event counting. Neuroreport 18(11):11571161. doi:10.1097/WNR.0b013e3281ace0ca

Bresciani J, Ernst M, Drewing K, Bouyer G, Maury V, Kheddar A (2005) Feeling what you hear: auditory signals can modulate tactile tap perception. Exp Brain Res 162(2):172-180. doi: 10.1007/s00221-004-2128-2

Bruce V, Young A (1998) In the eye of the beholder: the science of face perception. Oxford University Press, New York

Bruns P, Röder B (2010) Tactile capture of auditory localization: an event-related potential study. Eur J Neurosci 31(10):1844-1857. doi:10.1111/j.1460-9568.2010.07232.x

Bult JHF, de Wijk RA, Hummel T (2007) Investigations on multimodal sensory integration: texture, taste, and ortho- and retronasal olfactory stimuli in concert. Neurosci Lett 411(1):6-10. doi: 10.1016/j.neulet.2006.09.036

Bülthoff I, Newell FN (2006) The role of familiarity in the recognition of static and dynamic objects. Prog Brain Res 154:315-325. doi:10.1016/S0079-6123(06)54017-8

Bushnell EW, Baxt C (1999) Children's haptic and cross-modal recognition with familiar and unfamiliar objects. J Exp Psychol Hum Percept Perform 25(6):1867-1881

Casey SJ, Newell FN (2005) The role of long-term and short-term familiarity in visual and haptic face recognition. Exp Brain Res 166(3-4):583-591. doi:10.1007/s00221-005-2398-3

Casey SJ, Newell FN (2007) Are representations of unfamiliar faces independent of encoding modality. Neuropsychologia 45(3):506513. doi:10.1016/j.neuropsychologia.2006.02.011

Catherwood D (1993) The robustness of infant haptic memory: testing its capacity to withstand delay and haptic interference. Child Dev 64:702-710

Cattaneo Z, Vecchi T (2008) Supramodality effects in visual and haptic spatial processes. J Exp Psychol Learn Mem Cognit 34(3):631-642. doi:10.1037/0278-7393.34.3.631

Cattaneo Z, Vecchi T (2011) Blind vision: the neuroscience of visual impairment. MIT Press, London

Cattaneo Z, Vecchi T, Cornoldi C, Mammarella I, Bonino D, Ricciardi E, Pietrini P (2008) Imagery and spatial processes in blindness and visual impairment. Neurosci Biobehav Rev 32(8):1346-1360. doi:10.1016/j.neubiorev.2008.05.002

Chapman CE (1994) Active versus passive touch: factors influencing the transmission of somatosensory signals to primary somatosensory cortex. Can J Physiol Pharmacol 72(5): 558-570. NRC Research Press Ottawa, Canada. doi:10.1139/y94-080

Cholewiak RW, Collins AA, Brill C (2001) Spatial factors in vibrotactile pattern perception. In: Proceedings of the eurohaptics 2001, Birmingham, UK, 1-4 July 2001

Cinel C, Humphreys GW, Poli R (2002) Cross-modal illusory conjunctions between vision and touch. J Exp Psychol Hum Percept Perform 28(5):1243-1266. doi:10.1037//0096-1523.28.5.1243

Cohen H, Scherzer P, Viau R, Voss P, Lepore F (2011) Working memory for braille is shaped by experience. Commun Integr Biol 4(2):227-229. doi:10.4161/cib.4.2.14546

Corbetta D, Snapp-Childs W (2009) Seeing and touching: the role of sensory-motor experience on the development of infant reaching. Infant Behav Dev 32(1):44-58. doi:10.1016/j.infbeh.2008.10.004

Cornoldi C, De Beni R, Giusberti F, Massironi M (1998) Memory and imagery: a visual trace is not a mental image. In: Conway $\mathrm{M}$, Gathercole S, Cornoldi C (eds) Theories of memory, vol 2. Psychology Press, Hove, pp 87-110

Craddock M, Lawson R (2008) Repetition priming and the haptic recognition of familiar and unfamiliar objects. Percept Psychophys 70(7):1350-1365. doi:10.3758/PP.70.7.1350

Craddock M, Lawson R (2009a) Size-sensitive perceptual representations underlie visual and haptic object recognition. PLoS One 4(11). doi:10.1371/journal.pone.0008009
Craddock M, Lawson R (2009b) The effects of size changes on haptic object recognition. Atten Percept Psychophys 71(4):910-923. doi:10.3758/APP.71.4.910

Craig J, Evans P (1987) Vibrotactile masking and the persistence of tactual features. Percept Psychophys 42(4):309-317

Cronin V (1977) Active and passive touch at four age levels. Dev Psychol 13(3):253-256

Cruz A, Green BG (2000) Thermal stimulation of taste. Nature 403(6772):889-892. doi:10.1038/35002581

Delwiche J (2004) The impact of perceptual interactions on perceived flavor. Food Qual Prefer 15(2):137-146. doi:10.1016/S09503293(03)00041-7

Demattè ML, Sanabria D, Sugarman R, Spence C (2006) Cross-modal interactions between olfaction and touch. Chem Senses 31(4):291-300. doi:10.1093/chemse/bjj031

Deshpande G, Hu X, Lacey S, Stilla R, Sathian K (2010) Object familiarity modulates effective connectivity during haptic shape perception. NeuroImage 49(3): 1991-2000. Elsevier B.V. doi: 10.1016/j.neuroimage.2009.08.052

Easton R, Greene A, Srinivas K (1997a) Transfer between vision and haptics: memory for 2-D patterns and 3-D objects. Psychon Bull Rev 4(3):403-410

Easton R, Srinivas K, Greene A (1997b) Do vision and haptics share common representations? Implicit and explicit memory within and between modalities. J Exp Psychol Learn Mem Cognit 23:153-163

Ernst MO, Banks MS (2002) Humans integrate visual and haptic information in a statistically optimal fashion. Nature 415(6870): 429-433. doi:10.1038/415429a

Freides D (1974) Human information processing and sensory modality: cross-modal functions, information complexity, memory, and deficit. Psychol Bull 81(5):284-310

Gallace A, Spence C (2009) The cognitive and neural correlates of tactile memory. Psychol Bull 135(3):380-406. doi:10.1037/a0015325

Gallace A, Spence C (2010) The science of interpersonal touch: an overview. Neurosci Biobehav Rev 34(2):246-259. doi:10.1016/j. neubiorev.2008.10.004

Gallace A, Tan HZ, Haggard P, Spence C (2008) Short term memory for tactile stimuli. Brain Res 1190:132-142. doi:10.1016/j. brainres.2007.11.014

Garbin CP (1988) Visual-haptic perceptual nonequivalence for shape information and its impact upon cross-modal performance. J Exp Psychol Hum Percept Perform 14(4):547-553

Geffen G, Rosa V, Luciano M (2000a) Effects of preferred hand and sex on the perception of tactile simultaneity. J Clin Exp Neuropsychol 22(2):219-231

Geffen G, Rosa V, Luciano M (2000b) Sex differences in the perception of tactile simultaneity. Cortex 36(3):323-335

Gibson JJ (1962) Observations on active touch. Psychol Rev 69(6): 477-491. American Psychological Association. doi:10.1037/h004 6962

Gibson J (1966) The senses considered as perceptual systems. Houghton Mifflin, Oxford

Gillmeister H, Eimer M (2007) Tactile enhancement of auditory detection and perceived loudness. Brain Res 1160:58-68. doi: 10.1016/j.brainres.2007.03.041

Giudice NA, Betty MR, Loomis JM (2011) Functional equivalence of spatial images from touch and vision: evidence from spatial updating in blind and sighted individuals. J Exp Psychol Learn Mem Cognit 37(3):621-634. doi:10.1037/a0022331

Goodnow JJ (1971) Eye and hand: differential memory and its effect on matching. Neuropsychologia 9(1):89-95

Guclu B, Murat A (2007) Active touch does not improve sequential processing in a counting task. Acta Neurobiol Exp 67:165-169

Hagander L, Midani H, Kuskowski M, Parry G (2000) Quantitative sensory testing: effect of site and pressure on vibration thresholds. Clin Neurophysiol 111(6):1066-1069 
Hatwell Y (2003) Manual exploratory procedures in children and adults. In: Hatwell Y, Streri A, Gentaz E (eds) Touching for knowing. John Benjamins Publishing Company, Philadelphia

Helbig HB, Ernst MO (2008) Haptic perception in interaction with other senses. In: Grünwald M (ed) Human haptic perception: basics and applications, Birkhäuser Basel, Basel, pp 235-249. doi:10.1007/978-3-7643-7612-3_18

Heller M (1984) Active and passive touch: the influence of exploration time on form recognition. J Gen Psychol 110:243-249

Heller M, Ballesteros S (2006) Preface. In: Heller M, Ballesteros S (eds) Touch and blindness: psychology and neuroscience. Lawrence Erlbaum Associates, New Jersey

Heller MA, Jones ML, Walk AM, Schnarr R, Hasara A, Litwiller B (2010) Sex differences in the haptic change task. J Gen Psychol 137(1):49-62

Ho C, Tan HZ, Spence C (2006) The differential effect of vibrotactile and auditory cues on visual spatial attention. Ergonomics 49(7):724-738. doi:10.1080/00140130600589887

Homa D, Kahol K, Tripathi P, Bratton L, Panchanathan S (2009) Haptic concepts in the blind. Atten Percept Psychophys 71(4):690-698. doi:10.3758/APP.71.4.690

Hötting K, Röder B (2004) Hearing cheats touch, but less in congenitally blind than in sighted individuals. Psychol Sci 15(1):60-64

James TW, Blake R (2004) Perceiving object motion using vision and touch. Cogn Affect Behav Neurosci 4(2):201-207

Jones SE (1986) Sex differences in touch communication. West J Speech Commun Taylor \& Francis

Kaas A, Stoeckel M, Goebel R (2008) The neural bases of haptic working memory. In: Grunwald M (ed) Human haptic perception: basics and applications. Birkhäuser, Basel, pp 113-129

Kanwisher N (2000) Domain specificity in face perception. Nat Neurosci 3(8):759-763

Kappers AML, Douw F (2010) Hand movement investigations inspired by Yarbus. Perception, 39. Supplement ECVP conference abstracts, p 67

Kappers AML, Schakel WB (2011) Comparison of the haptic and visual deviations in a parallelity task. Exp Brain Res 208(3):467-473. doi:10.1007/s00221-010-2500-3

Katz D (1989) The world of touch (L. Krueger, Trans.). Lawrence Erlbaum, Hillsdale (original work published in 1925)

Kilgour A, Lederman SJ (2002) Face recognition by hand. Percept Psychophys 64(3):339-352. doi:10.3758/BF03194708

Kiphart MJ, Auday BC, Cross HA (1988) Short-term haptic memory for three-dimensional objects. Percept Mot Ski 66(1):79-91

Kiphart MJ, Hughes J, Simmons J, Cross HA (1992) Short-term haptic memory for complex objects. Bull Psychon Soc $30: 212-214$

Klatzky RL (1992) Stages of manual exploration in haptic object identification. Percept Psychophys 62:661-670

Klatzky RL, Lederman SJ (1995) Identifying objects from a haptic glance. Percept Psychophys 57(8):1111-1123

Klatzky RL, Lederman SJ (2003) The haptic identification of everyday life objects. In: Hatwell Y, Streri A, Gentaz E (eds) Touching for knowing. John Benjamins Publishing Company, Philadelphia

Klatzky RL, Lederman SJ, Metzger V (1985) Identifying objects by touch-an expert system. Percept Psychophys 37(4):299-302

Klatzky RL, Lederman SJ, Reed C (1987) There's more to touch than meets the eye: the salience of object attributes for haptics with and without vision. J Exp Psychol Gen 116(4):356-369

Klatzky RL, Loomis JM, Lederman SJ, Wake H, Fujita N (1993) Haptic identification of objects and their depictions. Perception Psychophysics 54:170-178

Lacey S, Campbell C (2006) Mental representation in visual/haptic crossmodal memory: evidence from interference effects. Q J Exp
Psychol (Colchester) 59(2):361-376. doi:10.1080/1747021050 0173232

Lacey S, Campbell C, Sathian K (2007) Vision and touch: multiple or multisensory representations of objects. Perception 36(10):15131521. doi:10.1068/p5850

Lacey S, Flueckiger P, Stilla R, Lava M, Sathian K (2010) Object familiarity modulates the relationship between visual object imagery and haptic shape perception. NeuroImage 49(3):19771990. doi:10.1016/j.neuroimage.2009.10.081

Laird DA (1932) How the consumer estimates quality by subconscious sensory impressions. J Appl Psychol 16(3): 241-246. American Psychological Association. doi:10.1037/h0074816

Lawson R (2009) A comparison of the effects of depth rotation on visual and haptic three-dimensional object recognition. J Exp Psychol Hum Percept Perform 35(4):911-930. doi:10.1037/ a0015025

Lawson R, Bulthoff HH (2008) Using morphs of familiar objects to examine how shape discriminability influences view sensitivity. Percept Psychophys 70(5):853-877. doi:10.3758/PP.70.5. 853

Lederman SJ, Klatzky RL (1987) Hand movements: a window into haptic object recognition. Cogn Psychol 19(3):342-368

Lederman SJ, Klatzky RL (1996) Action for perception: manual exploratory movements for haptically processing objects and their features. In: Wing A, Haggard P, Flanagan R (eds) Hand and brain: neurophysiology and psychology of hand. Academic, San Diego, pp 431-446

Lederman SJ, Klatzky RL (2004) Haptic identification of common objects: effects of constraining the manual exploration process. Percept Psychophys 66(4):618-628. doi:10.3758/BF03194906

Lederman SJ, Klatzky RL (2009) Haptic perception: a tutorial. Atten Percept Psychophys 71(7):1439-1459. doi:10.3758/APP.71. 7.1439

Lederman SJ, Loomis JM, Williams DA (1982) The role of vibration in the tactual perception of roughness. Percept Psychophys 32(2):109-116

Lederman SJ, Klatzky RL, Abramowicz A, Salsman K, Kitada R, Hamilton C (2007) Haptic recognition of static and dynamic expressions of emotion in the live face. Psychol Sci 18(2):158-164. doi:10.1111/j.1467-9280.2007.01866.x

Lhote M, Streri A (1998) Haptic memory and handness in 2-monthold infants. Laterality Asymmetries Bidy Brain Cognit 3(2):173-192

Loomis JM, Lederman SJ (1986) Tactual perception. In: Boff K, Kaufman L, Thomas J (eds) Handbook of perception and human performance, Organization, New York

Loomis JM, Klatzky RL, Lederman SJ (1991) Similarity of tactual and visual picture recognition with limited field of view. Perception 20(2): 167-177

Louw S, Kappers AML, Koenderink JJ (2002) Haptic discrimination of stimuli varying in amplitude and width. Exp Brain Res 146(1):32-37. doi:10.1007/s00221-002-1148-z

Luo Z, Imamiya A (2003) Color presentation mode affects human haptic memory for rough surfaces. In: Proceedings of the 2003 IEEE international conference on information reuse and integration, IRI-2003, Las Vegas, USA

Luo Z, Imamiya A (2004). Do colors affect our recognition memory for haptic rough surfaces. In: Computational science-Iccs 2004, Pt 3, proceedings, vol 3038, pp 897-904. Presented at the computational science-Iccs 2004, Pt 3, proceedings

Newell FN, Ernst MO, Tjan B, Bülthoff H (2001) Viewpoint dependence in visual and haptic object recognition. Psychol Sci 12(1):37

Norman JF, Norman HF, Clayton AM, Lianekhammy J, Zielke G (2004) The visual and haptic perception of natural object shape. Percept Psychophys 66(2):342-351 
Occelli V, Spence C, Zampini M (2008) Audiotactile temporal order judgments in sighted and blind individuals. Neuropsychologia 46(11):2845-2850. doi:10.1016/j.neuropsychologia.2008.05.023

Picard D (2006) Partial perceptual equivalence between vision and touch for texture information. Acta Psychol 121(3):227-248. doi:10.1016/j.actpsy.2005.06.001

Picard D, Lebaz S, Jouffrais C, Monnier C (2010) Haptic recognition of two-dimensional raised-line patterns by early-blind, lateblind, and blindfolded sighted adults. Perception 39(2):224-235. doi: $10.1068 / \mathrm{p} 6527$

Plaisier MA, Bergmann-Tiest WMBB, Kappers AML (2008) Haptic pop-out in a hand sweep. Acta Psychol 128(2):368-377. doi: 10.1016/j.actpsy.2008.03.011

Postma A, Zuidhoek S, Noordzij ML, Kappers AML (2007) Differences between early-blind, late-blind, and blindfoldedsighted people in haptic spatial-configuration learning and resulting memory traces. Perception 36(8):1253-1265

Pring L, Rusted J (1985) Pictures for the blind: an investigation of the influence of pictures on recall of text by blind children. Br J Dev Psychol. British Psychological Society

Richardson BL, Wuillemin DB, Mackintosh GJ (2011) Can passive touch be better than active touch? A comparison of active and passive tactile maze learning. Br J Psychol 72(3):353-362. doi: 10.1111/j.2044-8295.1981.tb02194.x

Röder B, Rösler F (2004) Compensatory plasticity as a consequence of sensory loss. In: Calvert G, Spence C, Stein B (eds) The handbook of multisensory processes. MIT Press, Cambridge, p 915

Sadato N, Ibanez V, Deiber M, Hallett M (2000) Gender difference in premotor activity during active tactile discrimination. NeuroImage 11(5):532-540. doi:10.1006/nimg.2000.0566

Sanabria D, Soto-Faraco S, Spence C (2005a) Spatiotemporal interactions between audition and touch depend on hand posture. Exp Brain Res 165(4):505-514. doi:10.1007/s00221-005-2327-5

Sanabria D, Soto-Faraco S, Spence C (2005b) Assessing the effect of visual and tactile distractors on the perception of auditory apparent motion. Exp Brain Res 166(3-4):548-558. doi: 10.1007/s00221-005-2395-6

Sanders AFJ, Kappers AML (2008) Factors affecting the haptic filledspace illusion for dynamic touch. Exp Brain Res 192(4):717-722. doi:10.1007/s00221-008-1635-y

Sathian K, Prather SC (2006) Cerebral cortical processing of tactile form: evidence from functional neuroimaging. In: Heller $M$, Ballesteros S (eds) Touch and blindness: psychology and neuroscience. Lawrence Erlbaum Associates, New Jersey

Sherrick CE (1975) The art of tactile communication. Am Psychol 30(3):353-360

Simões-Franklin C, Whitaker TA, Newell FN (2011) Active and passive touch differentially activate somatosensory cortex in texture perception. Hum Brain Mapp 32(7):1067-1080. doi: $10.1002 / \mathrm{hbm} .21091$

Spence C, Gallace A (2007) Recent developments in the study of tactile attention. Can J Exp Psychol/Revue Canadienne de Psychologie Expérimentale 61(3):196-207. doi:10.1037/cjep2007021

Spence C, Ho C (2008) Multisensory warning signals for event perception and safe driving. Theor Issues Ergon Sci 9(6): 523-554. Taylor and Francis Ltd. doi:10.1080/1463922070 1816765
Stadtlander LM, Murdoch LD, Heiser SM (1998) Visual and Haptic influences on memory: age differences in recall. Exp Aging Res 24(3):257-272

Stevens J, Foulke E, Patterson M (1996) Tactile acuity, aging, and braille reading in long-term blindness. J Exp Psychol Appl 2(2):91-106

Stier DS, Hall JA (1984) Gender differences in touch: an empirical and theoretical review. J Pers Soc Psychol 47(2): 440. American Psychological Association. doi:10.1037/0022-3514.47.2.440

Streri A, Lhote M, Dutilleul S (2000) Haptic perception in newborns. Dev Sci 3(3):319-327. doi:10.1111/1467-7687.00126

Titchener EB (1909) A text-book of psychology. New York: Macmillan

Trojan J, Getzmann S, Möller J, Kleinböhl D, Hölzl R (2009) Tactileauditory saltation: spatiotemporal integration across sensory modalities. Neurosci Lett 460(2):156-160. doi:10.1016/j.neulet. 2009.05.053

Turvey M (1996) Dynamic touch. Am Psychol 51(11):1134-1152

van der Horst BJ, Willebrands WP, Kappers AML (2008) Transfer of the curvature aftereffect in dynamic touch. Neuropsychologia 46(12):2966-2972. doi:10.1016/j.neuropsychologia.2008.06.003

Van Doorn GH, Richardson BL, Wuillemin DB, Symmons MA (2010) Visual and haptic influence on perception of stimulus size. Atten Percept Psychophys 72(3):813-822. doi:10.3758/APP.72.3.813

Verrillo RT (1968) A duplex mechanism of mechanoreception. In: Kenshalo D (ed) The skin senses. Thomas, Springfield

Verrillo RT, Gescheider GA (1975) Enhancement and sumation in the perception of two sucessive vibrotactile stimuli. Percept Psychophys 18:128-136

Wagner SH, Sakovits LJ (1986) A process analysis of infant visual and cross-modal recognition memory: implications for an amodal code. Adv Infancy Res. Elsevier/JAI Press

Weber E (1996) E. H. Weber on the tactile senses (H. Ross \& D. Murray, Trans.). Taylor \& Francis, Erlbaum (original works published in 1834 and 1905)

Whitaker TA, Simões-Franklin C, Newell FN (2008) Vision and touch: independent or integrated systems for the perception of texture. Brain Res 1242:59-72. doi:10.1016/j.brainres.2008.05.037

Willis F, Hofmann G (1975) Development of tactile patterns in relation to age, sex, and race. Dev Psychol 11(6):866

Woods AT, O'Modhrain S, Newell FN (2004) The effect of temporal delay and spatial differences on cross-modal object recognition. Cogn Affect Behav Neurosci 4(2):260-269

Woods AT, Moore A, Newell FN (2008) Canonical views in haptic object perception. Perception 37(12):1867-1878. doi:10.1068/ p6038

Zampini M, Brown T, Shore DI, Maravita A, Röder B, Spence C (2005) Audiotactile temporal order judgments. Acta Psychol 118(3):277-291. doi:10.1016/j.actpsy.2004.10.017

Zimler J, Keenan JM (1983) Imagery in the congenitally blind: how visual are visual images. J Exp Psychol Learn Mem Cogn 9(2):269-282

Zuidhoek S, Kappers AML, Postma A (2007) Haptic orientation perception: sex differences and lateralization of functions. Neuropsychologia 45(2):332-341. doi:10.1016/j.neuropsychologia.2 006.05.032 